\title{
A Critical Review Of The Basel Margin Of Conservatism Requirement In A Retail Credit Context
}

\author{
PJ de Jongh, Centre for BMI, North-West University, South Africa \\ T Verster, Centre for BMI, North-West University, South Africa \\ E Reynolds, Centre for BMI, North-West University, South Africa \\ M Joubert, Absa Retail Credit Risk, South Africa and PhD student, Centre for BMI, NWU \\ H Raubenheimer, Centre for BMI, North-West University, South Africa
}

\begin{abstract}
The Basel II accord (2006) includes guidelines to financial institutions for the estimation of regulatory capital (RC) for retail credit risk. Under the advanced Internal Ratings Based (IRB) approach, the formula suggested for calculating RC is based on the Asymptotic Risk Factor (ASRF) model, which assumes that a borrower will default if the value of its assets were to fall below the value of its debts. The primary inputs needed in this formula are estimates of probability of default (PD), loss given default ( $L G D)$ and exposure at default (EAD). Banks for whom usage of the advanced IRB approach have been approved usually obtain these estimates from complex models developed in-house. Basel II recognises that estimates of PDs, LGDs, and EADs are likely to involve unpredictable errors, and then states that, in order to avoid over-optimism, a bank must add to its estimates a margin of conservatism (MoC) that is related to the likely range of errors. Basel II also requires several other measures of conservatism that have to be incorporated. These conservatism requirements lead to confusion among banks and regulators as to what exactly is required as far as a margin of conservatism is concerned. In this paper, we discuss the ASRF model and its shortcomings, as well as Basel II conservatism requirements. We study the MoC concept and review possible approaches for its implementation. Our overall objective is to highlight certain issues regarding shortcomings inherent to a pervasively used model to bank practitioners and regulators and to potentially offer a less confusing interpretation of the MoC concept.
\end{abstract}

Keywords: Retail Credit Risk; Margin of Conservatism; Basel II; Measures Of Conservatism; Risk Factor Model

\section{INTRODUCTION}

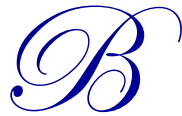

asel II is the second of the Basel Accords and contains recommendations on banking laws and regulations issued by the Basel Committee on Banking Supervision (BCBS, 2006). Of particular importance is the establishment of international standards that assist regulators in determining the capital that banks need to hold to guard against financial and operational risks. Basel II provides risk and capital management requirements in order to ensure that a bank has adequate capital for the risks it is exposed to as a result of its lending, investment and trading activities. In a retail credit risk context, Basel II recommends that regulatory capital (RC) be calculated by following either a standardised approach or an Internal Ratings Based (IRB) approach. Under the IRB approach, the formula suggested for calculating RC is based on the Asymptotic Risk Factor (ASRF) model which assumes that a borrower will default if the borrower's assets fall below the value of its debts. The ASRF model is based on the Merton (1974) model that was initially developed for the pricing of corporate debt. Vasicek (1977) showed that, under certain conditions, Merton's single asset model can be extended to model a portfolio of assets. The portfolio model used in the IRB approach (see e.g. Gordy (2003) bears a strong resemblance to Vasicek's model. The primary inputs needed in this regulatory capital (RC) formula are estimates of probability of default (PD), loss given default (LGD) and exposure at default (EAD). Banks who have obtained approval to use the advanced IRB approach usually obtain these estimates from complex models developed in-house. Basel II recognises that estimates of PDs, LGDs, and EADs are likely to involve unpredictable errors. It then states that, in order to avoid over-optimism, 
a bank must add to its estimates a margin of conservatism $(\mathrm{MoC})$ that is related to the likely range of errors. Basel II further requires that several other measures of conservatism have to be incorporated. These conservatism requirements lead to confusion among banks and regulators as to what exactly is required as far as margin of conservatism is required. In this paper we discuss the various conservatism requirements, investigate the $\mathrm{MoC}$ concept, and suggest possible approaches for its implementation. Overall, our objective is to bring certain shortcomings of pervasively used ASFR models to the attention of bank practitioners and regulators, in an attempt to clear up the confusion surrounding the $\mathrm{MoC}$, specifically from the South Africa viewpoint.

The layout of the report is as follows. Section 2 provides a brief description of the ASRF model as well as a discussion of some of its shortcomings. The Basel formula for calculating RC is reviewed and discussed. Then, in Section 3, some background is given regarding the conservatism requirements presented in the Basel documentation. This is followed in Section 4 by a discussion of the concept of $\mathrm{MoC}$ and the possible implementation thereof. Section 5 provides concluding remarks as well as comments on recent developments.

\section{THE ASRF MODEL AND REGULATORY CAPITAL}

\subsection{The RC Formula}

Regulatory capital (RC) is defined as the difference between the $99.9 \%$ VaR (Value at Risk) of the loss distribution and the expected loss. The expected loss must be covered by provisioning and the pricing of assets while RC should cover unexpected losses at the 99.9th percentile of the loss distribution (BCBS, 2005).

The regulatory capital formula prescribed by Basel II was derived in a wholesale context, and is based on the so-called Asymptotic Risk Factor (ASRF) model. This model is derived from an adaptation of the single asset Merton (1974) model. The ASRF model assumes that a borrower will default if the value of the borrower's assets falls below the value of its debts. The PD is derived as conditional on a systematic factor - a latent factor linked to asset value. The model assumes that changing assets are associated with this systematic factor as well as an idiosyncratic factor that depends on the borrower. The latent systematic factor represents the changing economic conditions (the economic cycle) which are universal to all portfolio assets. The systematic and the idiosyncratic factors are assumed to be independent and normally distributed. The PD distribution for a specific asset class then depends on the correlation between the assets of different borrowers as well as the estimated PDs obtained by some model or scorecard. The asset correlation $(\rho)$ is prescribed by Basel for different asset classes, e.g. for home loans $\rho=0.15$. The RC is linearly related to LGD and EAD and the difference between the expected PD and the $99.9 \% \mathrm{VaR}$ of the PD distribution. The ASRF model is discussed in detail in Van Gestel \& Baesens (2009), as well as in Aas (2005) and Annoli, Beccalli, \& Giordani (2013). Here we provide only the formula for calculating regulatory capital, and assume that the reader is familiar with the underlying assumptions and derivation of the model.

The formula for calculating RC is:

$$
\mathrm{RC}=(\mathrm{EAD})(\mathrm{LGD})\left[\Phi\left\{\left(\sqrt{\frac{\rho}{1-\rho}}\right) \Phi^{-1}(\mathrm{PD})+\left(\sqrt{\frac{\rho}{1-\rho}}\right) \Phi^{-1}(0.999)\right\}-\mathrm{PD}\right]
$$

where $\Phi($.$) is the standard normal distribution function. Note that the quantities PD, LGD and EAD have to be$ estimated, but $\rho$ is specified depending on the type of credit portfolio. For a loan portfolio, given estimates of PD, LGD and EAD for each obligor, the IRB capital charge can be computed by calculating capital charges for each loan (obligor), and then aggregating these to arrive at a portfolio capital charge see e.g. Van Gestel \& Baesens (2009) and Medema, Koning, Lensink, \& Medema (2009). Alternatively, as advocated by Thomas (2009) and Crook \& Bellotti (2010), given estimates of PD, LGD and EAD for each portfolio segment, RC can be calculated by aggregating the capital charges over segments. Here segments can be determined as a risk grade (based on PD bands) as suggested by Thomas (2009), or by other means using variables such as loan-to-value ratios. This approach is not viewed as a best practice, as it can be shown to understate capital as compared with obligor and loan level estimations, a consequence of the concavity of the Gordy formula and Jensen's inequality. Note that the estimation of especially LGD and EAD 
present special challenges in terms of explainability and predictability. These issues are discussed in detail in Jacobs \& Karagozoglu (2011) and Jacobs (2010).

\subsection{Shortcomings of the Model}

The RC formula is characterised by its computational simplicity; however, it has several weaknesses. Below we make some remarks about the underlying assumptions and possible limitations of this model:

- The model assumes that the bank's credit portfolio is 'infinitely fine-grained' in the sense that any single obligor represents a relatively small share of the portfolio's total exposure. The asymptotic RC formula was derived based on the assumption that all idiosyncratic risk is completely diversified away, which is rarely satisfied in practice, due to factors such as risk concentrations and the fact that certain obligors have larger exposures. Therefore the RC formula underestimates the capital because any portfolio of finite size carries some undiversified risk (Aas, 2005). This gave rise to the introduction of the so-called granularity adjustment to the Basel requirements. An improvement on the initial Basel granularity adjustment has been proposed, amongst others, by Emmer \& Tasche (2005).

- The model assumes a single underlying systematic risk factor. Such a model cannot reflect segmentation effects, and failure to recognise the diversification effects of segmentation could result in the overestimation of capital. In order to circumvent this problem, several authors have proposed multifactor models (see e.g. Fok, Yan, \& Yao (2014). However, depending upon the sensitivities of PD to the risk factors, the bias could go in the other direction.

- The model assumes that the LGD and EAD are both independent of the PD and more-over, that LGD and EAD are deterministic (non-stochastic). Several papers have provided evidence that dependence exists between loss events and loss given default (see e.g. Pykhtin (2003) and Eckert, Jakob, \& Fischer (2016). Also, since LGD and EAD are considered non-stochastic, one could argue that the RC formula underestimates the capital required to cover the unexpected losses. Although LGD and EAD are considered fixed in the original Merton framework, there is voluminous literature that relaxes this restriction, see e.g. Jacobs (2011).

- The different estimates of asset correlation $(\rho)$, as prescribed by Basel, have been criticised (see e.g. Miu \& Ozdemir (2006) and Sabato \& Schmid (2008). Although the process of determining these correlation estimates is described at a high level in an advisory note (BCBS, 2005), the precise details are not provided and the applicability to South African markets has not been established. General industry perception has been that these values may have been set too high.

- The model was originally designed for corporate loans, but the underlying assumptions may not apply to consumer credit. Not all borrowers wait until their total asset value falls below the value of their debt before defaulting. In particular, many defaulters are those who will not pay despite asset ownership, with evidence suggesting that cash-flow constraints affect default in the case of the individual, rather than overall asset value (see e.g. Annoli, Beccalli, \& Giordani (2013).

- The applicability of the normal distribution assumption for the systematic factor, representing the economic cycle, has been questioned in the literature. Lamb \& Perraudin (2008) suggest adding an assumption that the systematic factor follows an autoregressive process.

It is also interesting to note that RC is a linear function of LGD and EAD, but a strictly concave function of PD (see Van Gestel \& Baesens (2009). Figure 1 below illustrates this for different levels of LGD and PD when taking EAD = 1 and $\rho=0.15$. The RC increases up to a PD of about $11 \%$, after which it decreases. 
Figure 1. RC curves per unit of EAD as a function of PD and LGD at $\rho=0.15$.

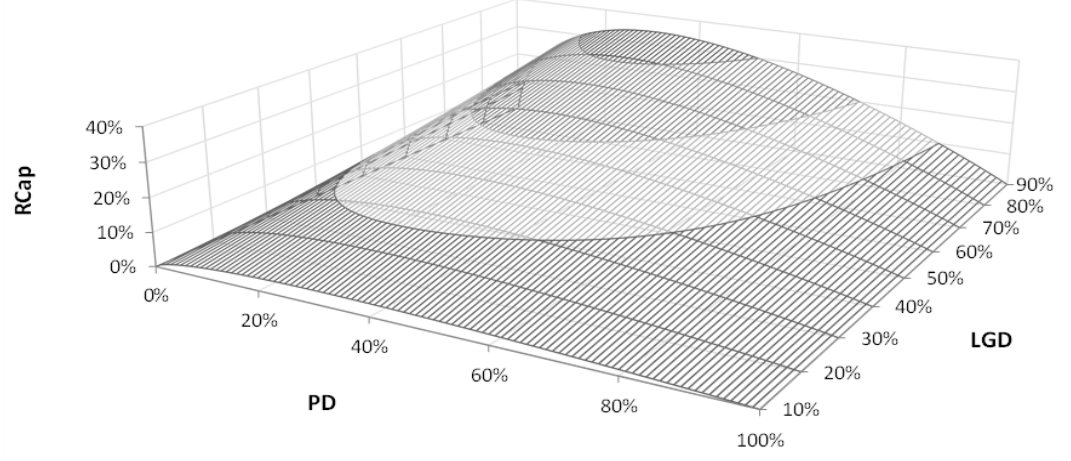

Table 1 below presents the PD at which the maximum RC would occur for a given asset correlation. This characteristic will be discussed in more detail in Section 4. For more detail on the behaviour of the RC formula, the interested reader is referred to Van Gestel \& Baesens (2009) and Thomas (2009).

We will now discuss briefly how the inputs for the RC formula may be obtained. We assume the reader will have knowledge of the inputs (PD, LGD, EAD) as described in e.g. Thomas (2009) and Anderson (2007). For practical guidelines, specifically in the course of implementing the Basel II framework, see OeNB/FMA (2004).

Table 1. PD at which the maximum RC is obtained as a function of asset correlation.

\begin{tabular}{c|c}
\hline Asset correlation $(\rho)$ & PD resulting in max RC \\
\hline 0.00 & 0.30 \\
\hline 0.01 & 0.25 \\
\hline 0.05 & 0.20 \\
\hline 0.10 & 0.15 \\
\hline 0.15 & 0.11 \\
\hline 0.20 & 0.10 \\
\hline 0.30 & 0.05 \\
\hline 0.40 & 0.03 \\
\hline 0.50 & 0.00 \\
\hline 0.60 & 0.00 \\
\hline
\end{tabular}

\subsection{Models for estimating PD}

The BCBS (2005) stipulates that the PD input into the RC formula should be so-called through-the-cycle PD (TTC PD) predictions. The TTC PD's should be estimated so that they reflect the expected default rate of an obligor over a period of time covering at least one full economic cycle. Technically, the PD is the centrality parameter of the default distribution, obtained through inverting the ASRF formula, so average default rates are only approximations to this. BCBS (2006) specifies that the PD inputs must be a long-run average of one-year default rates. The Federal Reserve in their Joint Final Rule pertaining to the Basel II Capital Accord (see Attachment 1, Board of Governors of the Federal Reserve System (2007), further specifies that this long-run average should be obtained over a mix of economic conditions that includes economic downturn conditions.

Using these PD's as input into the RC formula will lead to far less volatility in the RC estimate than the use of point in time PD's (PIT PD). Since PIT PD's assess the risk at a particular point in time, the borrower will move up or down rating grades through the economic cycle. Another approach would be the direct estimation of TTC PD's through structural models. In the basic ASRF model this is straightforward, and in many extensions not that much more computationally burdensome. It should be noted that under the basic ASRF model the long run default rates are good approximations. Some banks in South Africa have started modelling TTC PD directly. Under this approach, structural models are built by utilising macro-economic variables and non-cyclical risk drivers to predict default rates (TTC 
PD's) over an economic cycle (see e.g. Eckert, Jakob, \& Fischer (2016) for a corporate example of such a structural model). Data related to default rates and explanatory economic variables have to be collected over at least an entire economic cycle, and even if such data are available, challenges arise as a result of product changes and structural changes in the economic or financial system during the period under consideration (see e.g. Ingolfsson \& Elvarsson (2010).

Due to the above-mentioned difficulties it has become more popular to model the TTC PD by first estimating PIT PD for each portfolio segment and then transforming (or smoothing) this estimate to a TTC PD (see Thomas (2009). This approach is motivated by the fact that all banks have spent a considerable amount of time building and validating PIT PD models (see the paragraph below for more discussion on PIT PD models). The UK financial regulator, the Financial Services Authority (FSA), propagate the use of a variable scaling factor methodology in two memoranda, FSA (2007) and (2009). In this methodology, the transformation to a TTC PD is obtained by multiplying the PIT PD with a scalar. This scalar varies throughout the credit cycle. The FSA memoranda contain guidelines on the construction of variable scalars per segment (e.g. by risk grade), but since the guidelines provided are not explicit, many variants are possible. Following the guidelines in these documents, a local bank constructs $a$ so-called TTC scalar in a portfolio segment as the ratio of the average long run default rate and the average PIT PD at a specific point in time. For a particular segment, the average long run default rate is calculated by averaging the actual bad rate over an economic cycle (which must include a down-turn period), while the average PIT PD's are obtained as the average of the PIT PD estimates of the obligors in the particular segment. The TTC scalar is updated on a regular basis to ensure that the recent PIT PD is accurately scaled back to the long run average. Some banks implement this on a monthly basis, others bi-annually, creating a scalar that varies throughout the credit cycle. Of course, the definition of the start and end of the economic cycle is important, as is the definition of a downturn period.

Given the segment in which the obligor falls, the appropriate TTC scalar is then multiplied by the PIT PD of the obligor to obtain a TTC PD for the obligor. Clearly the TTC PD estimation approaches followed by different banks may vary, which makes regulatory control difficult. A more sophisticated methodology for calculating the TTC scalar has been proposed by Ingolfsson \& Elvarsson (2010). They use the Kalman filter to extract the economic cycle, which is then used to determine the appropriate scalar.

PIT PD is estimated by means of behavioural scorecards. The scorecard is usually built using logistic regression and a wealth of literature is available on its construction (see e.g. Siddiqi (2006), Anderson (2007) and Thomas (2009). A PIT PD is typically defined as the probability of an obligor (or account) defaulting in the following year, as estimated at a particular point in time. However, such a model could be estimated over a cycle, with repeated observations at a one year interval for each obligor, not only at a particular time period. These models are typically built based on data representing observed account statuses (default and non-default) for a particular year, by regressing this binary variable on explanatory variables lagged by one year. The PD predictions that result from these models are obtained at a specific point in time (usually a particular month), do not cover an entire economic cycle, and are typically more volatile than the TTC PD's. The so-called TTC scalar can therefore be regarded as a scalar that smooths PIT PD's to provide less volatile TTC PD's and therefore smooths out the cyclical economic effects contained in the PIT PD predictions. It may be necessary to calibrate PIT PD's in order to produce PD's in line with Basel II requirements, for example in terms of the definitions of default and time horizon and techniques used to construct the scorecard (see Anderson (2007) and Thomas (2009). Several calibration methods have been suggested in the literature (see e.g. Medema, Koning, Lensink, \& Medema (2009) and Glößner (2003).

\subsection{Models for Estimating LGD}

Yang \& Tkachenko (2012) outline some major differences between PD and LGD modelling. Default events are discrete events that follow a Bernoulli (or binomial) distribution, while LGD is a variable that typically follows a beta distribution in the interval $[0,1]$. The LGD is one minus the recovery rate, where the recovery rate is equal to the discounted recovered amount divided by the EAD. The LGD is therefore the loss fraction of the EAD, which will take several years after default to resolve, whereas PD describes the likelihood of a default event occurring in a specified period. In effect, LGD modelling is typically substantially more complex than PD (and EAD) modelling. The sources of complexity stem from, amongst others, the issue of developing the LGD measurements in line with Basel 
prescriptions, the paucity of data in some segments, gaps in risk driver data, the idiosyncratic nature of LGD and the challenge of explaining the idiosyncratic variability.

A distinction can be made between subjective and objective LGD methods (Engelman \& Rauhmeier, 2011). Subjective LGD methods make use of expert judgment and are used for low default portfolios, portfolios with insufficient data and new portfolios. Objective LGD methods can be classified into explicit (estimating LGD directly) and implicit methods (estimating LGD indirectly by using sub models). Explicit methods allow for the direct computation of LGDs, whereas with implicit methods LGD information can only be extracted by applying applicable procedures. Examples of explicit methods are the market LGD and the workout LGD approaches (see e.g. Hlawatsch \& Reichling (2010) while implied market LGD and implied historical LGD are classified as implicit methods (see Engelman \& Rauhmeier (2011). The market LGD and implied market LGD approaches are popular in a wholesale context and are not readily applicable in a retail context; therefore, we will only describe the workout LGD and the implied historical LGD approaches below.

The workout LGD is a cashflow-oriented explicit method and is typically applied when estimating LGD for unsecured retail products (Witzany, 2012). It has also been used for secured portfolios, where collateral information is used as explanatory variables for the LGD dependent variable. The workout LGD is equal to one minus the recovery rate, where the recovery rate can be calculated as the sum of all future recoveries, discounted to the default point and then expressed as a fraction of the exposure at default. Zhang \& Thomas (2012), Schmidt (2006) and Witzany (2012) respectively make use of a linear regression, run-off triangle and Cox proportional hazards regression to model the recovery rate directly. Focussing directly on the loss amount, Tong, Mues \& Thomas (2013) make use of a zero adjusted gamma model. In this case, LGD is calculated as the loss amount divided by the exposure at default.

The use of the implied historical LGD is only for retail portfolios that typically consist of secured products (Leow \& Mues, 2012). In their paper, Leow \& Mues (2012) make use of a combination of a haircut model and of a probability model to estimate the loss given default. This approach was followed by a local bank for their home loan portfolio. For written-off accounts, the bank estimated the shortfall by using a haircut model and used Cox-type survival models for estimating the probability of write-off given default and the probability of cure given default. Both these models were then combined to estimate the probability of curing, writing-off, or remaining incomplete. The challenges in empirically estimating the haircuts, and therefore in validating such models, have drawn regulatory scrutiny.

Because the focus of this paper is on the clarification of the MoC concept as opposed to a detailed exposition of LGD modelling techniques, the interested reader is referred to the papers by Lotheram, Brown, Martens, Mues \& Baesens (2012), Qi \& Zhao (2011), Bellotti \& Crook (2012), Leow \& Mues (2012) and Witzany (2012) for a comparison of LGD modelling techniques. However, the brief overview presented here does illustrate that LGD modelling may involve various approaches, some of which are quite complicated. As we will discuss further in Section 4 of this paper, complexity within any given modelling phase further complicates the process of isolating and reasonably quantifying MoC.

\subsection{Models for Estimating EAD}

Under the advanced IRB approach, banks are allowed to use their own estimates of EAD. Conceptually, EAD consists of two parts, namely the amount currently drawn, and an estimate of future drawdowns of available, but untapped credit (see e.g. Moral (2011) and Taplin, To, \& Hee (2007). Estimates of potential future drawdowns are known as credit conversion factors (CCFs). Since the CCF is the only random (unknown) proportion of EAD, the estimation of EAD ultimately amounts to the estimation of the CCF. The CCF is generally believed to depend on both the type of product and the type of borrower. The obligor risk rating has been found to also be a determinant, which will be closely related to type of borrower. At present, literature regarding these topics as well as appropriate data sources are in scarce supply. However, the BCBS (2005) does offer some suggestions as to which characteristics of the loan and the borrower should be taken into account in EAD estimation. Witzany (2011) offers a comprehensive review of various techniques for estimating EAD. He also provides a probabilistic definition of EAD, noting that it depends on the probability distribution of the time to default (specified as the time between a default date and a retrospective reference date), which in turn is determined by the nature of a particular product as well as time from facility origination. The 
EAD definition leads to the estimation of a "probability weighted conversion factor" by means of a regression approach.

The exposure at default (EAD) may be estimated by (see e.g. Yang \& Tkachenko (2012) and Taplin, To, \& Hee (2007):

$$
\mathrm{EAD}=\text { Outstanding Balance }+\mathrm{CCF} \times \text { Undrawn Limit }
$$

where Outstanding Balance is the account balance plus the accrued interest at a reference date, Undrawn Limit (Limit of the account minus the Outstanding Balance) is the amount available at the reference date and CCF (Credit Conversion Factor) is the proportion of the undrawn limit that is likely to be withdrawn in the event of default.

The CCF therefore represents the fact that if an account defaults in the future, the balance at default is expected to equal the balance today plus a fraction of the undrawn amount. The CCF on a non-defaulted facility is required to be non-negative and its estimation strongly depends on the time horizon. Since PD and LGD are considered at a one-year horizon, EAD should also be estimated as conditional upon default during the same one year horizon. There are several approaches, see e.g. Moral (2011), that aim to accomplish this, namely the cohort approach, the fixed time approach, the variable time approach, and more recently, the probability weighted approach (Witzany, 2011).

In some instances, the CCF is expressed in terms of the total credit limits (and not only out of the undrawn limit), and is then referred to as the loan equivalent factor (LEQ). Then the equation above changes to:

$$
\mathrm{EAD}=(\text { Outstanding Balance }+ \text { Undrawn Limit }) \times \mathrm{LEQ}
$$

The LEQ (Loan EQuivalent exposure) refers to the conversion factor on the total limit, i.e. the outstanding balance plus the undrawn limit (also sometimes referred to as the "Momentum approach"). In its simplest form, this equation does not fulfil the requirements of the capital adequacy requirements (Witzany, 2011), because it estimates the total-limit ratio rather than the CRD (Capital Requirements Directive) -compliant conversion factor, defined as a percentage of the undrawn amount (more details in CEBS (2006) paragraphs 299, 305, 306). This has the consequence that the currently drawn amount is not considered. However, according to CEBS (2006) the approach may be acceptable under specific situations (see e.g. paragraphs 306, 307 of CEBS (2006).

Local banks segment accounts based on limit utilization, making use of the LEQ for accounts with low or medium limit utilization and the CCF for accounts with high limit utilization. Conversion factors (CCFs and LEQs) are approximately one when the limit of the facility at reference date equals the exposure of the facility at reference date.

Several approaches have been proposed to estimate the conversion factors (see e.g. Taplin, To \& Hee, 2007 (2007) and Yang \& Tkachenko (2012). Local banks make use of a regression approach, similar to that described in Witzany (2011). The long run average CCF may be estimated by the slope of a simple regression of the "Outstanding Balance" at default (same as EAD) minus the "Outstanding Balance" at given reference dates against the "Undrawn Limit" at these reference dates.

\subsection{Summary of Review of ASRF}

The previous section has highlighted various issues with the RC formula and illustrated that a variety of different assumptions and methodologies are applied by practitioners when deriving the formula input. The statement from the advisory note regarding risk weights is also revealing: "It should be noted that the ASRF (Asymptotic Single Risk Factor) for use in the Basel risk weight functions does by no means express any preferences of the Basel Committee towards one model over others" (BCBS, 2005). The Basel regulations, therefore, do not enforce the use of the ASRF model. The South African Reserve Bank (SARB), however, requires that the RC formula is used for all RC calculations. 


\section{A SUMMARY OF BASEL CONSERVATISM REQUIREMENTS}

Due to recognition of the issues with the RC formula, as reviewed in the previous section, Basel (BCBS, 2006) introduced further regulatory requirements such as $\mathrm{MoC}$ and other measures of conservatism. In this section a summary of Basel conservatism requirements is provided. The relevant excerpts, listed in Table 2 below, have been classified as either pertaining directly to the MoC, or to other measures of conservatism that have to be incorporated during interim modelling phases.

Table 2. Excerpts from regulatory guidelines (BCBS, 2006; Board of Governors of the Federal Reserve System, 2013) regarding conservatism.

\begin{tabular}{|c|c|c|}
\hline Source & Relates to & Excerpt \\
\hline $\begin{array}{l}\text { Basel II, } \\
\text { Par } 451\end{array}$ & $\mathrm{MoC}$ & $\begin{array}{l}\text { In general, estimates of PDs, LGDs, and EADs are likely to involve unpredictable errors. } \\
\text { In order to avoid over-optimism, a bank must add to its estimates a MoC that is related to } \\
\text { the likely range of errors. Where methods and data are less satisfactory and the likely } \\
\text { range of errors is larger, the MoC must be larger. }\end{array}$ \\
\hline $\begin{array}{l}\text { Basel II, } \\
\text { Par } 468\end{array}$ & $\begin{array}{l}\text { Other } \\
\text { (LGD specific: } \\
\text { downturn, } \\
\text { correlation) }\end{array}$ & $\begin{array}{l}\text { LGD estimates should reflect economic downturn conditions and must equal or exceed the } \\
\text { long-run default-weighted average loss rate given default. } \\
\text { Banks are required to account conservatively for potential correlation between loss } \\
\text { severities and default probabilities. Banks are advised to base estimates on loss severities } \\
\text { observed during periods of high credit losses or to forecast based on conservative } \\
\text { assumptions. }\end{array}$ \\
\hline $\begin{array}{l}\text { Basel II, } \\
\text { Par } 475\end{array}$ & $\begin{array}{l}\text { MoC } \\
\text { (EAD specific) }\end{array}$ & $\begin{array}{l}\text { The EAD estimate must be an estimate of the long-run default-weighted average EAD for } \\
\text { similar facilities and borrowers over a sufficiently long period of time, but with a MoC } \\
\text { appropriate to the likely range of errors in the estimate. }\end{array}$ \\
\hline $\begin{array}{l}\text { Basel II, } \\
\text { Par } 475\end{array}$ & $\begin{array}{l}\text { Other } \\
\text { (EAD specific: } \\
\text { downturn) }\end{array}$ & $\begin{array}{l}\text { Moreover, for exposures for which EAD estimates are volatile over the economic cycle, } \\
\text { the bank must use EAD estimates that are appropriate for an economic downturn, if these } \\
\text { are more conservative than the long run average. }\end{array}$ \\
\hline $\begin{array}{l}\text { Basel II, } \\
\text { Par } 462\end{array}$ & $\begin{array}{l}\text { MoC } \\
\text { (PD specific) }\end{array}$ & $\begin{array}{l}\text { A bank may use data on internal default experience for the estimation of PD. A bank must } \\
\text { demonstrate in its analysis that the estimates are reflective of underwriting standards and } \\
\text { of any differences in the rating system that generated the data and the current rating } \\
\text { system. Where only limited data are available, or where underwriting standards or rating } \\
\text { systems have changed, the bank must add a greater MoC in its estimate of PD. The use of } \\
\text { pooled data across institutions may also be recognised. A bank must demonstrate that the } \\
\text { internal rating systems and criteria of other banks in the pool are comparable with its own. }\end{array}$ \\
\hline $\begin{array}{l}\text { Federal } \\
\text { Reserve: } \\
\text { BCC 13-5. }\end{array}$ & $\begin{array}{l}\text { Conservatism in } \\
\text { parameter } \\
\text { estimates }\end{array}$ & $\begin{array}{l}\text { The degree of conservatism applied to adjust for uncertainty should be related to factors } \\
\text { such as: } \\
\text { - the relevance of the reference data to bank's existing exposures, } \\
\text { - the robustness of the models, } \\
\text { - the amount of judgment used throughout the process. } \\
\text { Conservatism need not be additive for all elements of uncertainty. } \\
\text { A bank is not required to add a MoC at each step if doing so would produce an excessively } \\
\text { conservative result. Improvements in the quantification process (including the use of more } \\
\text { complete data and better estimation techniques) may reduce the appropriate degree of } \\
\text { conservatism over time. }\end{array}$ \\
\hline
\end{tabular}

Excerpts from Federal Reserve documents are included in light of the fact that US agencies encourage banks to develop estimates compliant with the requirements of the Advanced IRB approach, which could potentially lead to an insightful interpretation of the guidelines.

Furthermore, a conservative approach should be followed with respect to each of the sources of uncertainty indicated in Table 3 below. 
Table 3. Mapping of sources of uncertainty to Basel guidelines.

\begin{tabular}{l|l|l}
\hline Source of uncertainty & \multicolumn{1}{|c}{ Description } & \multicolumn{1}{|c}{ Basel framework paragraph } \\
\hline Data limitations & Adjustment to estimates are required to reflect data limitations & $411,460,462,475$ \\
\hline Estimation error & $\begin{array}{l}\text { Certain statistical models produce a point estimate for which the } \\
\text { variability is reflected in the width of the confidence interval. }\end{array}$ & 451,475 \\
\hline Expert judgment & $\begin{array}{l}\text { Own expertise (e.g. as related to workout and collection } \\
\text { experience) may influence estimates when empirical data or } \\
\text { applicable external data are not available. }\end{array}$ & 460,462 \\
\hline Operational changes & $\begin{array}{l}\text { Underwriting standards or rating systems may change over time, } \\
\text { making it difficult to directly compare data before and after the } \\
\text { change. }\end{array}$ & 462 \\
\hline Model risk & $\begin{array}{l}\text { Any model is an imperfect representation of reality. Over time, } \\
\text { more accurate or appropriate models may be developed or } \\
\text { identified. Model assumptions may fail to remain realistic under } \\
\text { certain conditions. }\end{array}$ & 451 \\
\hline Stress testing & $\begin{array}{l}\text { Estimates should reflect potential adverse economic conditions or } \\
\text { unexpected events. }\end{array}$ & $415,416,435$ \\
\hline Downturn calibration & $\begin{array}{l}\text { Estimates should reflect the impact of adverse economic } \\
\text { conditions or unexpected events similar to scenarios that have } \\
\text { occurred in the past or may occur in the future. }\end{array}$ & 468,475 \\
\hline Wrong-way risk & $\begin{array}{l}\text { Correlations between default risk and loss severities arising } \\
\text { during periods of stress should be incorporated into prediction } \\
\text { models where appropriate. }\end{array}$ & 468,475 \\
\hline
\end{tabular}

With reference to the extract provided in Table 4 below, it may be argued that the requirement for a MoC, especially in the case of PD, is unnecessary. The Value-at-Risk (VaR) for regulatory capital is $99.9 \%$, in effect guarding the bank against a 1 in a 1,000 year loss. In our opinion, deficiencies in the methodology for constructing a profit/loss distribution used to calculate RC are the root cause for many of the conservatism requirements and MoCs for PD, LGD and EAD.

Table 4. Excerpt from BIS (Bank for International Settlements) note regarding confidence levels.

\begin{tabular}{|c|c|c|}
\hline $\begin{array}{l}\text { BIS } \\
\text { Explanatory } \\
\text { Note } \\
\text { (BCBS. } \\
\text { 2005) }\end{array}$ & $\begin{array}{l}\text { IRB Risk } \\
\text { Weight } \\
\text { Functions }\end{array}$ & $\begin{array}{l}\text { The confidence level is fixed at } 99.9 \% \text {, i.e. an institution is expected to suffer losses } \\
\text { that exceed its level of Tier } 1 \text { and Tier } 2 \text { capital on average once in a thousand years. } \\
\text { This confidence level might seem rather high. However, Tier } 2 \text { does not have the loss } \\
\text { absorbing capacity of Tier } 1 \text {. The high confidence level was also chosen to protect } \\
\text { against estimation errors that might inevitably occur from banks' internal PD, LGD } \\
\text { and EAD estimation, as well as other model uncertainties. }\end{array}$ \\
\hline
\end{tabular}

\section{MARGIN OF CONSERVATISM}

\subsection{Introduction}

The intent of the Basel MoC requirement appears to be to encourage banks to amend estimates in order to:

- avoid over-optimism,

- $\quad$ reflect the "likely range of errors", and

- reflect uncertainty regarding models and data.

This is in addition to other measures of conservatism required to ensure that estimates reflect economic downturn and the potential for correlation across risk drivers. While it is clear that a MoC is required and that the requirement may be justifiable, a precise methodology for determining this margin is not definitively outlined in the Basel framework or associated literature for LGD or EAD (neither of which have an 'unexpected' component). The uncertainty that the MoC aims to address could include uncertainties encountered with the model inputs as well as with the model itself. In attempting to formulate a framework for deriving a suitable $\mathrm{MoC}$ based on the above statement of intent, the 
requirement potentially shows significant overlap with the objectives of guidelines for addressing model risk; see e.g. the statement contained in Table 4 (BCBS. 2005). The 2014 guideline of the Board of Governors of the Federal Reserve System in particular refers to the selection of appropriate goodness-of-fit criteria and the requirement that confidence intervals around estimated parameters should be explicitly documented (albeit from an operational risk perspective), while the 2011 document (Board of Governors of the Federal Reserve System, 2011) highlights the importance of model validation and on-going monitoring. The latter recommends: 'an effective challenge of models: critical analysis by objective, informed parties that can identify model limitations and produce appropriate changes'.

The Board of Governors of the Federal Reserve System (2013) in circular BCC 13-5 make some interesting remarks on the requirement for conservatism to the parameters in the advanced internal ratings based approaches: 'A bank is not required to add a margin of conservatism at each step if doing so would produce an excessively conservative result. Instead the overall margin of conservatism should adequately account for all uncertainties and weaknesses in the quantification process. Improvements in the quantification process, including the use of more complete data and better estimation techniques, may reduce the appropriate degree of conservatism over time'. This particular Federal Reserve document contains guidance to promote more extensive analysis of sources of uncertainty and more consistent methodological approaches for applying appropriately conservative parameter estimates across banking organisations. While the guiding principle is to treat each (input) parameter separately, there can be instances when an adjustment applied to one parameter suffices to address uncertainty across multiple parameters for a given portfolio. In such cases the justification for applying only the single adjustment should be documented and will be subject to additional supervisory scrutiny.

\subsection{Possible Statistical Approaches to MoC}

From a statistical point of view, two modelling approaches (referred to hereafter as A and B) are possible for estimating a MoC.

\section{Approach A}

The $100(1-\alpha)^{\text {th }}$ percentile of the empirical distribution of the prediction errors could be used, where the latter is obtained by comparing actual realisations with predicted values.

\section{Approach B}

The upper bound of the $100(1-\alpha) \%$ confidence interval for the predicted values could be used, usually obtained as standard output from statistical packages for most fitted models.

Because the historical prediction errors essentially form time series, an appropriate time series model should be used to analyse the data, unless the assumption of independence over time can be confirmed. For each approach, a particular value must be chosen for the confidence level $\alpha$, a choice which remains arbitrary unless more specific guidelines are provided. A further decision left to the discretion of the practitioner is the level at which the $\mathrm{MoC}$ concept should be applied, for instance: should such a margin be applied for every sub-model used, or should it be applied at an aggregate level? LGD models, for example, may comprise various sub-models; hence the modeller must decide if the MoCconcept is applicable to each model or only to the final model. The dependency structure between model outputs must also be considered and a decision must be made as to whether the MoCs applied to each model actually qualify as being additive or whether the assumption of additivity will result in unrealistic margins at the aggregate level. This is a complex consideration which, if addressed by means of further complex modelling, could lead to confusion and the inability to meaningfully interpret the final model output. Furthermore, the downside of this is underestimating MoC, as it may be doubtful that there is a diversification effect amongst component model errors. In our opinion, it should rather be applied at the highest level possible to ensure ease of interpretation.

The key to assigning the appropriate confidence level may potentially lie in the monitoring process where realised values can be monthly compared to model predictions. Based on Approach A, the real test would be to compare the predicted RC to the "realised RC", which will have to be based on RC estimates since actual RC (and LGD) can only be calculated for accounts that have been resolved, which in reality will only represent a subset of the full book. In 
this light the monitoring of actual versus predicted values will have to take place at the PD, LGD and EAD model level. The decision regarding the exact confidence level to employ should then be made based on the extent of the deviation between the actual (observed) and predicted values. In the light of these recommendations, the next three sections deal with the individual MoCs for PD, LGD and EAD.

\subsection{Formulation of MoC for PD}

In a retail context, we are yet to find a source that provides a clear methodology for constructing a $\mathrm{MoC}$ for the inputs into the $\mathrm{RC}$ formula. To this end, we suggest a formulation for constructing a MoC for PD's in general, and will use this as background for our further discussion of LGD and EAD.

For simplicity, only one risk grade is examined and all accounts in the risk grade are considered together. Let $p_{t}$ denote the actual observed default rate in period $t$ for $t=1,2, \ldots, n$. A default rate prediction model is used to determine predicted default rates $\hat{p}_{t}$, for $t=1,2, \ldots, n, n+1$. Rates are observed up to period $n$, but the model also supplies the predicted default rate $\hat{p}_{n+1}$ for the next period $n+1$. The problem to be dealt with is to adjust $\hat{p}_{n+1}$ so as to make it more conservative, but this is to be done in a justified manner since undue conservatism will have unwanted risk capital consequences. Let $l_{t}=\log \left(p_{t} /\left(1-p_{t}\right)\right.$ and $\hat{l}_{t}=\log \left(\hat{p}_{t} /\left(1-\hat{p}_{t}\right)\right.$ denote the log-odds transforms of the actual and predicted default rates. Then the adjustment of $\hat{l}_{n+1}$ is given by $\tilde{l}_{n+1}=\hat{l}_{n+1}+m$ where $m$ is the MoC to be used. The corresponding adjusted predicted default rate is $\tilde{p}_{n+1}=1 /\left(1+\exp \left(-\tilde{l}_{n+1}\right)\right.$. The outstanding requirement is a methodology for selecting the MoC. Let $e_{t}=l_{t}-\hat{l}_{t}$ for $t=1,2, \ldots, n$ denote the errors in the past predicted log-odds default rates when compared to the actual observed log-odds default rates. It is possible to fit a suitable time series model to the observed errors and to use this model to obtain a prediction $\hat{e}_{n+1}$ of the next error $e_{n+1}$.

\section{Approach A}

One possible approach would be to take $m=\hat{e}_{n+1}$, while an alternative would be a one-sided (upper) confidence bound for the next error, say $m=\hat{b}_{n+1}(\alpha)$ where the confidence level $\alpha$ can be selected to control the extent of conservatism. This suggestion is especially relevant when dealing with the PIT default prediction problem. The precise formulas used here would depend on the time series model fitted to the errors. If testing of the observed errors shows that they may be regarded as a random sample (i.e. no time dependence), this approach would be in line with taking $m=\hat{b}_{n+1}(\alpha)=100(1-\alpha)^{t h}$ percentile of the sample $e_{1}, \ldots, e_{n}$.

\section{Approach B}

If the prediction model used to generate the default rates $\hat{p}_{t}$ is sophisticated enough, it should be able to also supply confidence bounds for the $p_{t}{ }^{\prime}$ s. In particular, it should give a one-sided (upper) confidence bound, say $u_{n+1}(\alpha)$ for $p_{n+1}$, implying that the extent of conservatism can be controlled by selecting the confidence level $\alpha$. This modelling may be done via the $l_{t}$ 's, but the principle should be clear. The advantage is that only one model (presumably the model already implemented) is required, whereas modelling the errors in their own right would require an additional model with all the attendant issues regarding model selection to be dealt with. Even if Approach B is implemented, a study of the error series may be valuable. For example, a consistently positive (or negative) error series would indicate strongly that the basic prediction model has flaws that need to be addressed. Again, if the error series tends to become smaller (larger) over time, the basic prediction model must be improving (deteriorating) and this has implications for the $\mathrm{MoC}$ required.

As stated in Section 2.3, Basel requires that TTC PD estimates be used as an input to the RC formula. The most popular methodology currently followed by banks is the variable scalar approach in accordance with the guidelines given in FSA (2007) and (2009). In this methodology, two quantities are being estimated, namely the so-called TTC scalar and the PIT PD's. Should MoC's then be constructed for the TTC scalars as well as for the PIT PD's? Although the FSA memoranda provide guidelines on the choice of economic cycle, measures of conservatism to be incorporated, data issues and model validation, no clear guidelines are given to answer this question. 
As mentioned in Section 2.3, one of the banks in South Africa uses a specific implementation of this scalar approach. The TTC PD per segment is adjusted upwards by taking the $95^{\text {th }}$ percentile of observed default rates. This could be interpreted as more of a stringent conservatism requirement than a $\mathrm{MoC}$ as defined above. Judging by the requirements of FSA (2007) close judgmental monitoring of the scaling framework will always be required. Statistically rigorous tests can be designed and implemented at each re-estimation point of the scalar to flag suspicious portfolio developments and to avoid mistakenly incorporating permanent effects into the cycle estimate (Ingolfsson \& Elvarsson, 2010). In fact, this is what the supervisor expects most banks to do, at least for some years after implementing a variable scalar methodology.

In our opinion, if the FSA guidelines are followed and adequate attention is given to the specification of conservatism (such as calculating the TTC scalar using a down-turn period) and continuous model validation and monitoring (such as comparing actual default rates with PIT PD's), no MoC needs to be incorporated on the PD inputs to the RC formula. This is because the RC formula already makes provision for unexpected PD's and model uncertainty, which is not the case for LGD's and EAD's. Also, the cyclical nature of PIT PD estimates and their volatility further complicates the construction of a MoC. Rather, as suggested by the FSA, the effort should be spent on ensuring the accuracy of the PIT models via regular monitoring and validation and then, if required, model recalibration or redevelopment. Furthermore, as noted in Section 2.2 the RC formula is convex in PD. Suppose we are dealing with a home loan portfolio and our methodology recommends the application of a MoC to an estimated TTC PD of $11 \%$. If the margin results in a PD that is higher than $11 \%$, the resulting RC will be lower, instead of higher as intuitively expected. Although a higher PD would still contribute to the overall capital set aside (in the form of provisions, as determined by a higher expected losses) this finding is interesting in the context of regulatory capital, raising potential questions regarding the cost associated with holding regulatory capital (set aside annually) as opposed to provisions (amended on a month-to-month basis). In the context of a MoC, seen as a factor by which the regulatory capital is increased, this finding suggests that the MoC should only be applied up to a threshold of $11 \%$ or not at all. Again, we believe the answer lies in monitoring the error series continuously (as confirmed by the guidelines given by the FSA (2009) as well as the Board of Governors of the Federal Reserve System (2013) in circular BCC 13-5).

Branco (2011) proposes a model validation methodology for assessing the MoC included in PD estimates. This methodology can be seen as a benchmark from which comparisons can be drawn rather than an attempt to quantify the margin of conservatism. As a benchmark, the methodology compares, in each risk grade, the average losses recorded (historical information) and the estimated average losses, with the latter obtained from credibility premiums. Branco (2011) concludes that differences between estimated and recorded losses should be interpreted as a warning sign of possible mismatches in the margin of conservatism included in each risk grade, but should not lead, per se, to an automatic adjustment in PD estimations. Branco (2011) furthers states that the assessment of the margin of conservatism included in PD estimates cannot be isolated from the characteristics of the sample (in terms of nature, availability, timeliness and representativeness) nor from the inherent assumptions regarding calibration, business cycle, role of borrower dependencies and results of stress-testing exercises.

\subsection{Formulation of MoC for LGD}

The RC capital formula assumes that the loss given default rate (which is equal to one minus the recovery rate) is known and non-stochastic (Gordy, 2003). It should be noted that during an economic downturn, losses on defaulted loans are likely to be higher than under ordinary business conditions. As a single example, it is likely that the value of collaterals may decline. In this light, Basel II requires that a "downturn" LGD be estimated for each client/risk segment, but do not provide a specific methodology for doing so. Some banks might use a scalar to estimate the downturn LGD (similar to the PD TTC scalar approach). Alternatively, a bank can estimate the LGD (directly or indirectly) using data from a downturn period.

Banks are required to provide their own LGD estimates. These LGD estimates will consist of both an average LGD for expected loss related to defaulted assets and the performing book as well as a "downturn" LGD for expected losses and RC estimation. Sabato \& Schmid (2008) also address the issue of estimating downturn LGD. They note that the use of downturn LGD is recommended in the Basel Accord for the express purpose of compensating for possible PDLGD correlation that would otherwise not be accounted for when implementing the ASRF model. 
A further regulatory requirement for retail home loans is a $10 \%$ floor for the LGD estimate. In the home loan portfolio of a local bank, this LGD floor requirement contributes almost $75 \%$ of the total RC estimate. In this example, a MoC for a secured portfolio would have been unnecessary, given the regulatory floor. Given newly proposed floors, significant portions of the book may fall below the prescribed minimum level. As a result, $\mathrm{MoC}$ has little bearing on risk weighted assets. The newly suggested floors can be found in Table 2 of the consultative paper published by the BCBS (2016). In other situations, MoC might be introduced based on a lower or upper prediction confidence interval for the specific statistical model used to estimate LGD (e.g. linear regression, survival models). Note than whenever confidence intervals are employed the underlying model assumptions, e.g. the assumption of independent observations, must be tested thoroughly.

As stated previously, a MoC may be viewed as a post-modelling add-on to account for over-optimism, uncertainty regarding data, models or estimation error, over and above other conservative measures such as the explicit Basel requirement that the estimate should reflect downturn conditions. The adjustment of the downturn LGD scalar, if discrepancies are observed between predicted and actual values, may serve as a robust method for ascertaining whether or not the implemented MoCs are realistic.

Peter (2011) makes some remarks about MoCs in an LGD context. A too conservative view on downturn assessments is argued, as well as the use of empirical distributions and bootstrapping to determine quantiles selected for the MoC. Sabato \& Schmid (2008) propose a technique in the form of stress-testing for deriving conservative LGD estimates where banks lack extensive historical LGD data. The approach applies stressing factors to the cure rate and collateral value observed across risk-homogenous loan-to-value (LTV) pools, after which the migration of clients into higher LTV pools (due to deteriorating collateral value) is assessed in order to calculate a "stressed LGD". The approach results in specific adjustment formulae for each asset class. Although the bank may not necessarily lack historical data relevant to a credit event, this approach may be considered as a benchmark for comparison (Sabato \& Schmid, 2008).

In our view, since actual LGDs do not exist for incomplete accounts, Approach B should be used for constructing a MoC for the estimated LGD. This should be compared with the stressed downturn LGD estimate and the most conservative option taken.

\subsection{Formulation of MoC for EAD}

It is a Basel requirement that the conversion factors be modelled in a conservative way. Basel requires that either long run conversion factor estimates with a MoC or downturn estimates must be used for regulatory capital determination. In order to make logical sense, all $E A D$ estimates are determined by requiring that conversion factors to be floored at zero. At account level the maximum of the $E A D$ estimate and the Outstanding Balance is taken as the EAD estimate.

When considering the construction of a MoC for the EAD input to the $\mathrm{RC}$ formula, the focus is on the estimation of the CCF's. Downturn estimates of the CCF may be calculated using the downturn cycles used for the PD and LGD model development. Estimates of long run conversion factors with a MoC may be compared with downturn conversion factor estimates to determine the estimates for use in the final EAD RC estimation. Moral (2011) proposes a robust quantile regression procedure for determining conservative long run conversion factors for all portfolio segments. Witzany (2011) investigates an alternative approach for estimating CCF and gives an explicit expression for calculating a MoC for the resulting CCF. Witzany (2011) proposes the use of a $95 \%$ confidence interval around the regression coefficient CCF estimate, on the basis that a reference period spanning years with both high and low observed PD's would lead to an estimate capturing both estimation error and systemic variation due to economic

downturn. For reference periods that are not considered representative of the full spectrum of possible PD values, an unspecified additional conservative adjustment based on expert judgment or external data is recommended.

Once again, we believe that the stressed downturn EAD estimate should be compared with the MoC, using Witzany's suggestion, and the most conservative option taken. 


\section{CONCLUDING REMARKS}

A number of regulatory (Basel II) enforced conservative measures have to be incorporated by banks in their retail credit models (BCBS, 2006). Above and beyond these measures, it is also required in Basel II (BCBS, 2006) that banks should include MoC's for PD, LGD and EAD models. It should be clear that the myriad of conservatism requirements, along with an unclear methodology for the implementation of the MoC concept, contributes to a great deal of uncertainty among banks (and the regulator).

We have argued that the PD estimates do not require the MoC add-on and that for LGD and EAD, the maximum of the $\mathrm{MoC}$ and stressed downturn estimate be used. Also, it is very important to monitor, on a monthly basis, the performance of PD, LGD and EAD models over time. This could give the necessary indication as to when models need to be recalibrated or when the confidence levels used for the construction of MoCs for LGD and EAD need to be adjusted.

On-going sensitivity analyses to study the effects of using different $\mathrm{MoC}$ techniques (such as prediction intervals for survival curves) and different choices of confidence levels of the existing MoC measures are also encouraged. This is confirmed by the Board of Governors of the Federal Reserve System (2013) in circular BCC 13-5, stating "In general, conservatism should be applied as a temporary measure while actions are taken to develop more adequate data and empirical support. Accuracy and reliability remain the primary goal of the risk-parameter segmentation and quantification process." and also "Conservatism is not a substitute for taking actions to address identified problems in risk-measurement processes or data".

The main point of criticism is that the Vasicek model is not an ideal model for modelling retail credit risk losses and is therefore also not appropriate for estimating RC. However, the Basel Committee does not require that this specific model be used, and the local regulator could ostensibly be persuaded to employ a more appropriate approach.

In March 2016, the Basel Committee on Banking Supervision revisited the use of IRB approaches in the calculating risk weighted assets related to credit risk (BCBS, 2016). The proposed changes to the IRB approaches include a number of complementary measures that aim to firstly reduce the complexity of the regulatory framework and improve comparability; and secondly to address excessive variability in the capital requirements for credit risk. Specifically, the Basel Committee proposes to:

- remove the option to use the IRB approaches for certain exposures, where it is judged that the model parameters cannot be estimated sufficiently reliably for regulatory capital purposes;

- adopt exposure-level, model-parameter floors to ensure a minimum level of conservatism for portfolios where the IRB approaches remain available; and

- provide greater specification of parameter estimation practices to reduce variability in risk-weighted assets (RWA) for portfolios where the IRB approaches remain available.

However, if these proposals are implement, the question arises whether banks will continue to develop and utilise internal models as an internal risk management tool without the regulatory imperative. Without the freedom to use their own models in the hope that increased accuracy could lead to reduced capital requirements, it is possible that banks will see less of a need to do their own modelling for risk purposes, resulting in decreasing levels of understanding of the risks they bear (Hastings, 2016). This is a further critique of the MoC requirement, i.e. slavish conservatism may be related to lackadaisical modelling practices and less effective risk management.

It is interesting that the International Financial Reporting Standard (IFRS) called the IFRS9 Accord (IFRS, 2014) is moving towards a more complex approach for calculating expected loss (moving from an incurred to an expected loss methodology), whereas the Basel framework (BCBS, 2016) is now moving to a simpler standardised approach. We would like to underscore the importance of ensuring consistency between the accounting and prudential frameworks in the light of the IFRS9 (2014) accounting norms. For example, the loss distribution considered for calculating provisions (IFRS 9, based on expected losses), should be the same one as for calculating regulatory capital (Basel III, based on unexpected losses). If different definitions are used for expected losses, it will become increasingly difficult if not impossible for regulators and banks to make sense of the conflicting requirements. This paper has highlighted 
several issues when models with shortcomings are used to calculate regulatory capital. As soon as the model shortcomings are realised, ad hoc measures are introduced to cater for these deficiencies, ultimately giving rise to a number of sometimes conflicting requirements that make it difficult for country regulators to enforce and for banks to implement in a clear cut way.

\section{ACKNOWLEDGEMENTS}

We gratefully acknowledge the valuable contributions by Gary van Vuuren and Hennie Venter that led to improvements in this paper.

This work is based on research supported in part by the Department of Science and Technology (DST) of South Africa. The grant holder acknowledges that opinions, findings and conclusions or recommendations expressed in any publication generated by DST-supported research are those of the author(s) and that the DST accepts no liability whatsoever in this regard.

\section{AUTHOR BIOGRAPHIES}

Riaan de Jongh is the Director of the Centre for Business Mathematics and Informatics at the North-West University (NWU). He has received awards for the best published research in a particular year from the Operational Research Society of South Africa (ORSSA), the South African Statistical Association (SASA), the Actuarial Society of South Africa (ASSA) and Risk.net. He is a Past President and Fellow of SASA, received a Thought Leader Award from SASA and a National Recognition award from ORSSA. He also served on the boards of several local and global associations. He is the Vice-Chairperson of the NWU Pension Fund and served on various committees for the Global Association of Risk Professionals, the Professional Risk Managers' International Association, the International Society for Business and Industrial Statistics, and the Global Certified Enterprise Risk Actuary (CERA) Review Panel.

Tanja Verster is an associate professor at the Centre for Business Mathematics and Informatics at North-West University, South Africa, began her career with a Master's degree in Quantitative Risk Management. After working at First National Bank as a quantitative analyst, she continued her career at the North-West University as a lecturer in May 2003. She completed her PhD in Risk Analysis (focussing on credit scoring) in the Centre for BMI in 2007 and currently lectures post-graduates in courses ranging from credit scoring and predictive modelling to data mining. She is also involved in applied research projects (focusing on predictive modelling in the credit scoring environment).

Elzabe Reynolds is a quantitative risk analyst at Rand Merchant Bank (RMB), the investment banking arm of the First Rand group. She joined the Fixed Income, Currencies and Commodities devision immediately after obtaining her Master's degree in quantitative risk management from North-West University in 2008. In 2012, she returned to the university as a lecturer at the Centre for BMI, in subjects related to financial statistics. She rejoined RMB in 2016 and is currently a member of the Global Markets Risk Insights team, investigating enterprise-wide risk concerns raised by the chief risk officer.

Morne Joubert is a Senior Manager Retail Credit Basel and IFRS 9 model development at ABSA, South Africa. He has a Master's degree in Mathematical Statistics and is currently studying towards a PhD in Risk Analysis (focusing on LGD model development) at the Centre for Business Mathematics and Informatics at North-West University, South Africa. He began his career as a scorecard developer at Standard Bank where he later managed over the regulatory capital model development team. His industry experience extends over 10 years, focusing on managing regulatory and impairment model development teams.

Helgard Raubenheimer is an associate professor and Head of the SASLab and BMI professional masters programmes at the Centre for BMI, North-West University. He received a Ph.D. in Risk Analysis from the NorthWest University. His main research interest is in quantitative risk management. He has also supervised several master degree students on their industry research projects and is involved in research and consultation projects for financial institutions. He is also co-author of numerous peer-reviewed papers, which have received national and global awards. 


\section{REFERENCES}

Aas, K. (2005). The basel II IRB approach for credit portfolios: A survey. Note No SAMBA/33/05. Norsk Regnesentral, SAMBA/33/05.

Anderson, R. (2007). The credit scoring toolkit: Theory and practice for retail credit risk management and decision automation. Oxford: Oxford University Press.

Annoli, M., Beccalli, E., \& Giordani, T. (2013). Retail credit risk management. Palgrave Macmillan Studies in Banking And Financial Institutions.

BCBS. (2005, July). An explanatory note on the Basel II IRB Risk Weight Functions. Bank for International Settlements.

BCBS. (2006). Basel II: International convergence of capital measurement and capital standards: A revised framework. Bank for International Settlements.

BCBS. (2016). Reducing variation in credit risk-weighted assets - constraints on the use of internal based approaches. Bank for International Settlements.

Bellotti, T., \& Crook, J. (2012). Loss given default models incorporating macroeconomic variables for credit cards. International Journal of Forecasting, 28, 171-182.

Board of Governors of the Federal Reserve System. (2013). Applying the requirement for conservatism to the parameters in the advanced approaches (BCC 13-5). Basel Coordination Committee, Division of Banking Supervision and Regulation, Washington, D.C.

Board of Governors of the Federal Reserve System. (2007). Joint final rule and supporting board documents: risk-based capital standards. Advanced Capital Adequacy Framework-Basel II. Washington, DC.

Board of Governors of the Federal Reserve System. (2011). Guidance on Model Risk Management (SR 11-7). Washington, D.C.

Board of Governors of the Federal Reserve System. (2014). Supervisory guidance for data, modeling, and model risk management (BCC 14-1). Washington, D.C.

Branco, C. (2011). Addressing the issue of conservatism in probability of default estimates: a validation tool. The Journal of Risk Model Validation, 5(3), 3-19.

CEBS. (2006). Guidelines on the implementation, validation and assessment of Advanced Measurement (AMA) and Internal Rating Based (IRB) approaches, CP 10 Revised. London: Committee of European Banking Supervisors.

Charaborty, G., Pagolu, M., \& Garla, S. (2013). Text mining and analysis: Practical methods, examples, and case studies using SAS. SAS Institute.

Crook, J., \& Bellotti, T. (2010). Time varying and dynamic models for default risk in consumer loads. Journal of the Royal Statistical Society: Series A, 173(2), 283-305.

Eckert, J., Jakob, K., \& Fischer, M. (2016). A credit portfolio framework under dependent risk parameters: Probability of default, loss given default and exposure at default. Journal of Credit Risk, 12(1), 97-119.

Emmer, S., \& Tasche, D. (2005). Calculating credit risk capital charges with the one-factor model. Journal of Risk, 7(2), 85-101.

Engelman, B., \& Rauhmeier, R. (2011). The Basel II Risk Parameters: Estimation, validation, and stress testing. (second edition) (2nd ed.). Berlin: Springer.

Fok, P. W., Yan, X., \& Yao, G. (2014). Analysis of credit portfolio risk using hierarchical multifactor models. Journal of Credit Risk, 10(4), 45-70.

FSA. (2007, July 30). Use of variable scaling factors to derive long run probabilities of default for Retail portfolios. Memorandum to the Credit Risk Standing Group. Retrieved April 13, 2016, from UK Financial Supervisory Authority: http://www.fsa.gov.uk/pubs/international/crsg_variable_scalars.pdf

FSA. (2009, February 19). Variable scalar approaches to estimating through the cycle PDs. Memorandum to the Credit Risk Standing Group. Retrieved April 13, 2016, from UK Financial Supervisory Authority: http://www.fsa.gov.uk/pubs/international/variable_scalars.pdf

Glößner, P. (2003). Calculating Basel II Risk Parameters for a portfolio of retail loans. Thesis submitted in partial fulfilment of the requirements for an MSc. in Mathematical Finance, Kellogg College, Oxford University.

Gordy, M. (2003). A risk-factor foundation for risk-based capital rules. Journal of Financial Intermediation, 12, $199-232$.

Hastings, S. (2016). The end of the affair. The Observer Column, Commissioning Editor: Sarah Hastings: Email newsletter received 5 May 2016 of @Risk Books.

Hlawatsch, S., \& Reichling, H. (2010, Spring). A framework for loss given default validation of retail portfolios. The Journal of Risk Model Validation, 4(1), 23-48.

IFRS. (2014). IRFS9 Financial Instruments. International Accounting Standards Board.

Ingolfsson, S., \& Elvarsson, B. T. (2010). Cyclical adjustment of point-in-time PD. Journal of Operational Research Society, 61, 374-380.

Jacobs, J. M. (2010). An empirical study of exposure at default. The Journal of Advanced Studies in Finance, 1(1), 31-59.

Jacobs, J. M. (2011). A two-factor structural model of ultimate loss-given-default: capital structure and calibration to corporate recovery data. The Journal of Financial Transformation, 31, 31-43.

Jacobs, J. M., \& Karagozoglu, A. (2011). Modeling ultimate loss given default on corporate debt. The Journal of Fixed Income, 21(1), 6-20.

Lamb, R., \& Perraudin, W. R. (2008). Dynamic default rates. Imperial College Working Papers: TBS/RML/WP11. 
Leow, M., \& Mues, C. (2012). Predicting loss given default (LGD) for residential mortgage loans: A two-stage model and empirical evidence for UK bank data. International Journal of Forecasting, 28, 183-195.

Lotheram, G., Brown, I., Martens, D., Mues, C., \& Baesens, B. (2012). Benchmarking regression algorithms for loss given default modelling. International Journal of Forecasting, 28, 161-170.

Medema, L., Koning, R. H., Lensink, R., \& Medema, M. (2009). A practical approach to validating a PD model. Journal of Banking \& Finance., 33(4), 701-708.

Merton, R. C. (1974). On the pricing of corporate debt: The risk structure of interest rates. Journal of Finance, 2, 49-71.

Miu, P., \& Ozdemir, B. (2006). Basel requirements of downturn loss given default: Modeling and estimating probability of default and loss given default correlations. Journal of Credit Risk, 2(2), 43-68.

Moral, G. (2011). EAD estimates for facilities with explicit limits. In B. Engelmann, \& R. Rauhmeier (Eds.), The Basel II Risk Parameters (pp. 201-246). Berlin Heidelberg: Springer-Verlag.

OeNB/FMA. (2004). Guidelines on credit risk management - Rating models and validation. Austria: Oesterreichische Nationalbank (OeNB) \& Financial Market Authority (FMA).

Peter, C. (2011). Estimating loss given default: Experience from banking practice. In B. Engelmann, \& R. Rauhmeier (Eds.), The Basel II Risk Parameters (pp. 151-182). Berlin Heidelberg: Springer-Verlag.

Pykhtin, M. (2003). Unexpected recovery risk. RISK, 16, 74-79.

Qi, M., \& Zhao, X. (2011). Comparison of modelling methods for Loss Given Default. Journal of Banking \& Finance, 35, $2842-$ 2855.

Sabato, G., \& Schmid, M. (2008). Estimating conservative loss given default. ABN AMRO, Group Risk Management.

Schmidt, K. (2006). Methods and models of loss reserving based on run-off triangles: A unifying survey. Fall 2006. Casualty Actuarial Society Forum.

Siddiqi, N. (2006). Credit Risk Scorecards: Developing and Implementing Intelligent Credit Scoring. New Jersey: John Wiley \& Sons.

Taplin, R., To, H. M., \& Hee, J. (2007). Modeling exposure at default, credit conversion factors and the Basel II Accord. Journal of Credit Risk, 3(2), 75-84.

Thomas, L. C. (2009). Consumer credit models: Pricing, profit and portfolios. Oxford: Oxford University Press.

Tong, E., Mues, C., \& Thomas, L. (2013). A zero-adjusted gamma model for mortgage loan loss given default. International Journal of Forecasting, 29, 548-562.

Van Gestel, J., \& Baesens, B. (2009). Credit risk management: Basic concepts. Oxford: Oxford University Press.

Vasicek, O. (1977). An equilibrium characterization of the term structure. Journal of Financial Economics, 5, 177-188.

Witzany, J. (2011). Exposure at default modeling with default intensities. European Financial and Accounting Journal, 6(4), 2048.

Witzany, J. (2012). Survival analysis in LGD modeling. European Financial and Accounting Journal, 7(1), 6-27.

Yang, B. H., \& Tkachenko, M. (2012). Modeling exposure at default: Empirical approaches and technical implementation. Journal of Credit Risk, 8(2), 81-102.

Zhang, J., \& Thomas, L. (2012). Comparisons of linear regression and survival analysis using single and mixture distributions approaches in modelling LGD. International Journal of Forecasting, 28(1), 204-215. 


\section{NOTES}

